Ergod. Th. \& Dynam. Sys. (1989), 10, 287-294

Printed in Great Britain

\title{
Homotopical stability of pseudo-Anosov diffeomorphisms
}

\author{
ALBERT FATHI \\ The Institute for Advanced Study† and CNRS UA 1169, Université Paris-Sud, France
}

(Received 15 March 1988)

\begin{abstract}
We show that the pseudo-Anosov diffeomorphisms have a kind of stability even outside their own homotopy class, this generalizes some results of Lewowicz and Handel. As a corollary, we show that two pseudo-Anosov maps, with the same dilatation coefficient, which are semi-conjugate on the $\pi_{1}$ level are also semiconjugate as dynamical systems by a map which is a ramified cover.
\end{abstract}

Our main interest in this work is to find when a dynamical system $g: N \rightarrow N$ on a compact connected space can be semi-conjugated onto a pseudo-Anosov map. This is reminiscent of J. Franks work [F], it is also related to work of J. Lewowicz [L] and $M$. Handel [H1, H2].

A pseudo-Anosov diffeomorphism $f$ of a surface $M$ is a homeomorphism, for which there exists a pair of transverse measured foliations $\left(\mathscr{F}^{s}, \mu^{s}\right),\left(\mathscr{F}^{u}, \mu^{u}\right)$ and $\lambda>1$ such that $f_{*}\left(\mathscr{F}^{s}, \mu^{s}\right)=\left(\mathscr{F}^{s}, \lambda^{-1} \mu^{s}\right)$ and $f_{*}\left(\mathscr{F}^{u}, \mu^{u}\right)=\left(\mathscr{F}^{u}, \lambda \mu^{u}\right)-$ see [FLP] for Thurston's theory of measured foliations and pseudo-Anosov diffeomorphisms. In the beginning, our work was motivated by an attempt to classify pseudo-Anosov maps up to ramified covers, because we wanted to find out the smallest surface on which a given pseudo-Anosov diffeomorphism was 'living' naturally. Our Theorem 2 below shows that this can be reduced to an algebraic semi-conjugacy problem on the fundamental group level. When M. Handel's paper [H1] appeared, it was clear that this problem was related to a form of dynamical 'stability' for pseudo-Anosov diffeomorphisms which is the content of our Theorem 1 below. In fact, the proof of Theorem 1 produces more than its statement, we find a natural hyperbolic extension of the pseudo-Anosov map in which the surface sits naturally as the smallest non-trivial invariant compact connected subset. The space on which the hyperbolic extension exists has a universal cover which is the product of the two trees obtained from the stable and unstable foliations.

The following theorem generalizes some work of J. Lewowicz [L] and M. Handel [H1].

THEOREM 1. Let $f: M \rightarrow M$ be a pseudo-Anosov map of the closed connected surface

† Supported by NSF Grant No. DMS-8610730(1). 
$M$. Let $g: N \rightarrow N$ be a homeomorphism of the compact connected space $N$. Suppose that $\alpha: N \rightarrow M$ is a continuous map such that the diagram:

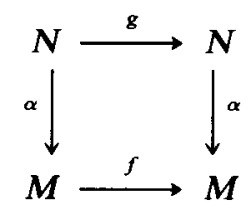

commutes up to homotopy. If $\alpha$ is not homotopic to a constant, then there exists a closed subset $Y \subset N$, which is $g$ invariant, and a continuous surjective map $\beta: Y \rightarrow M$ such that $\beta$ is homotopic to $\alpha \mid Y$ and the following diagram commutes:

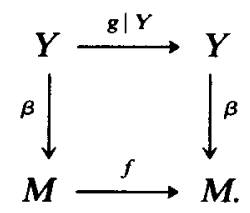

The second theorem is a generalization of the fact that two homotopic pseudoAnosov maps are conjugate, it should also be compared with [H2].

THEOREM 2. Let $f: M \rightarrow M$ and $g: N \rightarrow N$ be pseudo-Anosov maps, with the same dilatation coefficient on the closed connected surfaces $M$ and $N$. Suppose that $\alpha_{*}: \pi_{1}(M) \rightarrow \pi_{1}(N)$ is a non constant algebraic homomorphism such that the following diagram commutes:

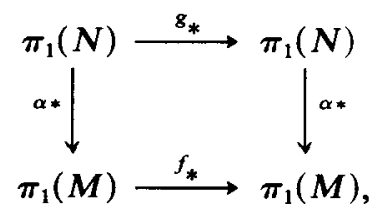

where $f_{*}$ and $g_{*}$ are respectively representatives of the action of $f$ and $g$ on the fundamental groups. Then there exists a ramified cover $\alpha: N \rightarrow M$ which semi-conjugates $g$ to $f$, and whose action on fundamental groups can be represented by $\alpha_{*}$.

\section{Some facts about trees and measured foliations}

Let $M$ be a closed connected surface and let $\mathscr{F}$ be a minimal measured foliation on $M$. Call $\tilde{M}$ the universal cover of $M$. Denote by $\tilde{F}$ the pullback of $\mathscr{F}$ to $\tilde{M}$. We call a leaf of $\tilde{F}$ either a regular leaf which does not contain a separatrix or the union of a singularity and all separatrices ending in that singularity. Since $F$ is minimal, any such leaf is a closed subset of $\tilde{M}$. We call $\mathscr{T}$ the set of leaves $\tilde{M} / \tilde{\mathscr{F}}$. If we define the distance between two leaves of $\tilde{F}$ as the minimum of the transverse measures of arcs joining the two leaves, we obtain a distance on $\mathscr{T}$ which turns $\mathscr{T}$ into a tree - see Morgan and Shalen [MS, $\S 2$ ]. If the genus of $M$ is $\geq 2$, then $\mathscr{T}$ is not complete for this distance. We will denote by $\hat{\mathscr{T}}$ its completion, it is also a tree, see [MS, proof of Theorem 2.1.9]. To simplify notations, we will denote by $\Gamma$ the 
fundamental group of $M$ the group $\Gamma$ acts in a natural way as a group of isometries of $\mathscr{T}$ and hence it also acts as a group of isometries of $\hat{\mathscr{T}}$.

LEMMA 1.1. If $a$ is a closed arc contained in $\hat{\mathscr{T}}$ then a minus its endpoints is contained in $\mathscr{T}$. If $f \in \mathscr{T}$, the set $\hat{\mathcal{T}} \backslash \Gamma f$ is totally disconnected.

In order to prove 1.1 , we need a couple of sublemmas.

Sublemma 1.2. Let $\alpha$ (resp. $\alpha^{\prime}$ ) be an arc between $x$ and $y$ (resp. $x^{\prime}$ and $y^{\prime}$ ) in a tree. The intersection $\alpha \cap \alpha^{\prime}$ is an arc of length l( $\left.\alpha \cap \alpha^{\prime}\right)$ satisfying:

$$
l\left(\alpha \cap \alpha^{\prime}\right) \geq l(\alpha)-d\left(x, x^{\prime}\right)-d\left(y, y^{\prime}\right) .
$$

The proof of this sublemma can be found in [MS, proof of Corollary 2.1.7].

Sublemma 1.3. Let $\hat{\mathscr{T}}$ be the completion of the tree $\mathscr{T}$. If $\alpha$ is an arc in $\hat{\mathscr{T}}$, then $\alpha$ minus its extremities is contained in $\mathscr{T}$.

Proof. Call $x$ and $y$ the extremities of $\alpha$. Choose $x_{n} \rightarrow x$ and $y_{n} \rightarrow y$ with $x_{n}, y_{n} \in \mathscr{T}$. Call $\alpha_{n} \subset \mathscr{T}$ the arc between $x_{n}$ and $y_{n}$. We have $\alpha \cap \alpha_{n} \subset \mathscr{T}$ and $l\left(\alpha \cap \alpha_{n}\right) \rightarrow l(\alpha)$ by 1.2 . The sublemma follows easily.

Proof of 1.1. Since the foliation $\mathscr{F}$ is minimal and any non trivial arc in $\mathscr{T}$ contains the image under the map $\tilde{M} \rightarrow \mathscr{T}$ of a non trivial arc transverse to $\tilde{F}$, we obtain that any non trivial arc of $\mathscr{T}$ contains a point of $\Gamma f$. By 1.2 , the same is true for any non trivial arc in $\hat{\mathfrak{T}}$. Lemma 1.1 follows from this last fact.

\section{Embedding a pseudo-Anosov map in a hyperbolic dynamical system}

Let $f: M \rightarrow M$ be a pseudo-Anosov map. Let $\lambda>1$ be its dilatation coefficient and let $\mathscr{F}^{s}$ and $\mathscr{F}^{u}$ be its stable and unstable foliations. Call $\left(\mathscr{T}^{u}, d^{u}\right)$ and $\left(\mathscr{T}^{s}, d^{s}\right)$ the trees $\tilde{M} / \tilde{F}^{s}$ and $\tilde{M} / \tilde{F}^{u}$ with their respective metrics. Fix a lift $\tilde{f}$ of $f$ to $\tilde{M}$. This $\tilde{f}$ induces actions $\tilde{F}^{u}$ and $\tilde{F}^{s}$ on the trees $\mathscr{T}^{u}$ and $\mathscr{T}^{s}$ which satisfy:

$$
\begin{aligned}
& \forall a, a^{\prime} \in \mathscr{T}^{u}, \quad d^{u}\left(\tilde{F}^{u}(a), \tilde{F}^{u}\left(a^{\prime}\right)\right)=\lambda d^{u}\left(a, a^{\prime}\right) \\
& \forall b, b^{\prime} \in \mathscr{T}^{s}, \quad d^{s}\left(\tilde{F}^{s}(b), \tilde{F}^{s}\left(b^{\prime}\right)\right)=\lambda^{-1} d^{s}\left(b, b^{\prime}\right) .
\end{aligned}
$$

This implies that these actions extend to the completions $\hat{\mathscr{T}}^{u}$ and $\hat{\mathscr{T}}^{s}$ and that these extensions verify the same equalities.

If we look at the product action $\tilde{F}=\tilde{F}^{s} \times \tilde{F}^{\mu}$ on $\tilde{Z}=\hat{\mathscr{T}}^{s} \times \hat{\mathscr{T}}^{u}$ with the product metric $d=d^{s}+d^{u}$ we obtain what we can call a metrically split hyperbolic homeomorphism - generalizing to arbitrary metric spaces [F, Definition on p. 67].

There is an inclusion $\tilde{M} \hookrightarrow \tilde{Z}$, which is obtained by sending a point in $M$ to the pair of leaves - one from $\tilde{F}^{s}$ and the other from $\tilde{\mathscr{F}}^{u}$ - that contain it. It is well known that the metric $d$ induces on $\tilde{M}$ the usual topology.

The action of $\Gamma=\pi_{1}(M)$ on $\tilde{M}$ induces isometries on $\tilde{Z}$ in a natural way. The inclusion $\tilde{M} \hookrightarrow \tilde{Z}$ is equivariant with respect to these actions of $\Gamma$.

If we write $\tilde{f} \gamma=f_{*}(\gamma) \tilde{f}$ for $\gamma \in \Gamma$, we also have $\tilde{F} \gamma=f_{*}(\gamma) \tilde{F}$. 
LEMMA 2.1. There exists $\varepsilon>0$ such that:

$$
\forall \gamma \in \Gamma \backslash\{\mathrm{Id}\}, \quad \forall z \in \tilde{Z}, d(\gamma z, z) \geq \varepsilon .
$$

Proof. If $z=\left(a^{u}, a^{s}\right) \in \tilde{\mathscr{F}}^{u} \times \tilde{F}^{s}$ then $\gamma z=\left(\gamma a^{u}, \gamma a^{s}\right)$. This implies:

$$
\begin{aligned}
d(\gamma z, z) & =d^{u}\left(\gamma a^{u}, a^{u}\right)+d^{s}\left(\gamma a^{s}, a^{s}\right) \\
& \geq i\left(\gamma, \mathscr{F}^{u}\right)+i\left(\gamma, \mathscr{F}^{s}\right) .
\end{aligned}
$$

It is well known that there exists $\varepsilon>0$ such that:

$$
\forall \gamma \in \Gamma \backslash\{\mathrm{Id}\}, i\left(\gamma, \mathscr{F}^{u}\right)+i\left(\gamma, \mathscr{F}^{s}\right) \geq \varepsilon .
$$

COROllary 2.2. The action of $\Gamma$ on $\tilde{Z}$ is properly discontinuous.

If we call $Z=\tilde{Z} / \Gamma$, we obtain a metric space since $d$ is $\Gamma$ equivariant. The map $\tilde{Z} \rightarrow Z$ appears as the universal cover of $Z$ since $\tilde{Z}$ is contractible and locally contractible. The inclusion $\tilde{M} \hookrightarrow \tilde{Z}$ gives an inclusion $M \hookrightarrow Z$ which is an isomorphism on the fundamental group. The map $\tilde{F}$ gives a map $F: Z \rightarrow Z$ which is a hyperbolic homeomorphism whose universal cover is metrically split. The images of the foliations $\left\{\hat{\mathscr{T}}^{s} \times b \mid b \in \hat{\mathscr{T}}^{u}\right\}$ and $\left\{a \times \hat{\mathscr{T}}^{u} \mid a \in \hat{\mathscr{T}}^{s}\right\}$ under the map $\tilde{Z} \rightarrow Z$ give the stable and unstable foliations of $F$. The restriction of $F$ to $M$ is $f$.

Lемма 2.3. In the situation described above, two continuous maps $g_{1}, g_{2}: X \rightarrow M$ are homotopic as maps with values in $M$ if and only if they are homotopic as maps with values in $Z$.

Proof. Since the genus of the surface $M$ is $\geq 1$, we can endow $M$ with a Riemannian metric such that any pair of points in the universal cover $\tilde{M}$ can be connected by a unique geodesic. Given any path $\alpha \subset Z$ connecting the two points $x, y \in M$, a lift $\tilde{\alpha}$ to $\tilde{Z}$ connects two points $\tilde{x}, \tilde{y} \in \tilde{M}$, call $\alpha^{\prime}$ the image in $M$ of the unique geodesic connecting $\tilde{x}, \tilde{y}$ in $\tilde{M}$. The map $\alpha \mapsto \alpha^{\prime}$ is well defined and continuous in the compact open topology. The lemma follows routinely from this fact.

LEMMA 2.4. If $x \in M$, then $Z \backslash\left(W^{s}(x, F) \cup W^{u}(x, F)\right)$ is totally disconnected.

Proof. Choose a point $\left(f, f^{\prime}\right) \in \tilde{Z}$ above $x$. We have $f \in \mathscr{T}^{s}, f^{\prime} \in \mathscr{T}^{u}$. It follows from 1.1 that $\left(\hat{\mathscr{T}}^{s} \backslash \Gamma f\right) \times\left(\hat{\mathscr{T}}^{u} \backslash \Gamma f^{\prime}\right)$ is totally disconnected. But this product is precisely the inverse image of $Z \backslash\left(W^{s}(x, F) \cup W^{u}(x, F)\right)$ under the covering map $\tilde{Z} \rightarrow Z$.

Proposition 2.5. If $X$ is a closed connected non empty subset of $Z$ which is $F$ invariant then either it is reduced to a point or it contains $M$.

Proof. Suppose that $X$ is not reduced to a point. Since the periodic points of $f=F \mid M$ are dense in $M$ and $X$ is closed, it suffices to show that $X$ contains these periodic points. Fix such a periodic point $p$. Since $X$ is closed and invariant under $F$, it suffices to show that either $W^{s}(p, F)$ or $W^{u}(p, F)$ intersects $X$. But this follows clearly from 2.4 , since $X$ is connected and not reduced to a point.

The next theorem is a generalization of Theorem 1 . 
THEOREM 2.6. Suppose that we have a diagram:

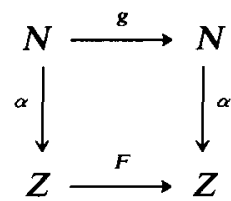

commutative up to homotopy, where $g$ is a homeomorphism of the compact connected space $N$. Then there exists a continuous map $\beta: N \rightarrow Z$, homotopic to $\alpha$, and such that the diagram:

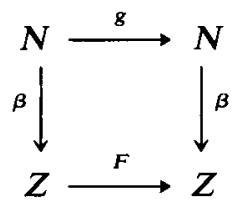

commutes. Moreover, if $\alpha$ is not homotopic to a constant, then the image $\beta(N)$ contains M. It follows that $Y=\beta^{-1}(M)$ is invariant under $g$ and that $\beta \mid Y$ is a continuous surjection onto $M$, which is a semi-conjugacy between $g \mid Y$ and $f$. Moreover, if $\alpha(Y) \subset M$ then $\alpha \mid Y$ and $\beta \mid Y$ are homotopic as maps with values in $M$.

Proof. Since $Z$ has a universal cover on which $F$ has a hyperbolic metrically split lift with a complete metric. It is easy to see that the machinery developed by $J$. Franks in [F, \& 4] can be applied to give $\beta: N \rightarrow Z$, homotopic to $\alpha$ which gives a semi-conjugacy:

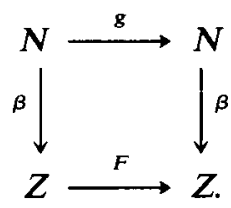

If $\alpha$ is not homotopic to a constant, by 2.3 , the image $\beta(N)$ is a compact connected $F$ invariant subset of $Z$ which is not reduced to a point. By 2.5 , we have $M \subset \beta(N)$. The last assertion follows from 2.3 .

3. Pseudo-Anosov diffeomorphisms with the same dilatation coefficient The following lemma is certainly well known.

LEMMA 3.1. Let $g: N \rightarrow N$ be a pseudo-Anosov diffeomorphism on a closed connected surface, with dilatation coefficient $\lambda>1$. Suppose that $Y \subset N$ is a compact $g$-invariant subset, if the topological entropy of $g \mid Y$ is $\log \lambda$, then $Y=N$.

Proof. From [FLP, Exposé $10 \S$ IV and $\S$ VI], there exists an irreducible subshift of finite type $\left(\Sigma_{A}, \sigma_{A}\right)$, whose topological entropy is $\log \lambda$, and a surjective semiconjugacy $\theta: \Sigma_{A} \rightarrow N$ between $\sigma_{A}$ and $g$. The closed subset $\theta^{-1}(Y)$ is $\sigma_{A}$-invariant and the topological entropy of $\sigma_{A}$ restricted to that subset is $\log \lambda$, it follows from [CP, Theorem 3.3] that $\theta^{-1}(Y)=\Sigma_{A}$. The surjectivity of $\theta$ implies that $\boldsymbol{Y}=\mathbf{N}$. 
Let $f: M \rightarrow M$ and $g: N \rightarrow N$ be pseudo-Anosov maps, with the same dilatation coefficient $\lambda$ on the closed connected surfaces $M$ and $N$. Suppose that $\alpha_{*}: \pi_{1}(N) \rightarrow$ $\pi_{1}(M)$ is a non constant algebraic homomorphism such that the following diagram commutes:

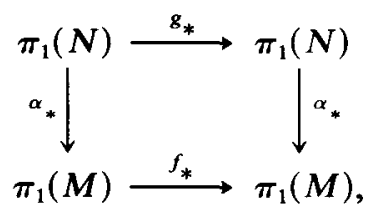

where $f_{*}$ and $g_{*}$ respectively represent $f$ and $g$ on the fundamental group.

The first remark is that there exists a map $\bar{\alpha}: N \rightarrow M$, which can be represented by $\alpha_{*}$ on $\pi_{1}$ and such that the diagram:

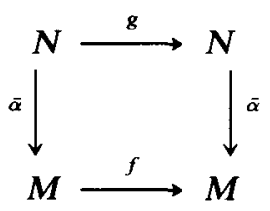

commutes up to homotopy. These are standard facts from algebraic topology. By Theorem 2.6, there exists $Y \subset N$ a $g$-invariant subset and a surjective semi-conjugacy $\alpha: Y \rightarrow M$, between $g \mid Y$ and $f$, with $\alpha$ homotopic to $\bar{\alpha} \mid Y$. Since the topological entropy of both $f$ and $g$ is $\log \lambda$, the topological entropy of $g \mid Y$ is also $\log \lambda$. By 3.1, we have $Y=N$ and, in fact, the map $\alpha$ is a surjective semi-conjugacy between $g$ and $f$, which is homotopic to $\bar{\alpha}$. The following lemma finishes the proof of Theorem 2.

LeMMA 3.2. Any non constant semi-conjugacy between two pseudo-Anosov maps, with the same dilatation coefficient on closed connected surfaces is a ramified cover..

Proof. Let us denote by $\varphi: N \rightarrow M$ a non constant semi-conjugacy between the two pseudo-Anosov diffeomorphisms $g: N \rightarrow N$ and $f: M \rightarrow M$ with the same dilatation coefficient $\lambda$. We denote by $\left(\mathscr{F}^{s}, \mu^{s}\right),\left(\mathscr{F}^{u}, \mu^{u}\right)$ the invariant stable and unstable measured foliations of $f$ and by $\left(\mathscr{G}^{s}, \nu^{s}\right),\left(\mathscr{G}^{u}, \nu^{u}\right)$ those of $g$. Since $\varphi$ is a semiconjugacy it takes a leaf of $\mathscr{G}^{s}$ (resp. $\mathscr{G}^{u}$ ) to a leaf of $\mathscr{F}^{s}$ (resp. $\mathscr{F}^{u}$ ).

What we mean by a segment contained in a $\mathscr{G}^{u}$ leaf is a subset of a $\mathscr{G}^{u}$ leaf which is homeomorphic to $[0,1]$ and if it contains a singularity then it must be entirely contained in the union of the singularity and of two separatrices adjacent to the same sector. Remark that the holonomy along $\mathscr{G}^{s}$ is always defined on one side of such a segment. If $\alpha$ is a segment in a $\mathscr{G}^{u}$ leaf, we denote by $\nu^{s}(\alpha)$ its $\nu^{s}$ measure and by $\bar{\mu}^{s}(\alpha)$ the measure of the $\mathscr{F}^{u}$ segment which has the same extremities as $\varphi(\alpha)$. It is clear that $\bar{\mu}^{s}$ is continuous on its domain of definition endowed with the compact open topology. Since $g$ and $f$ have the same dilatation coefficient $\lambda$, we have:

$$
\forall \alpha \mathscr{G}^{u} \text {-segment } \bar{\mu}^{s}(f(\alpha))=\lambda \bar{\mu}^{s}(\alpha) .
$$

If $\gamma$ is a segment of a $\mathscr{G}^{u}$ leaf which is the union of the two subsegments $\alpha$ and $\beta$ 
then we have:

$$
\bar{\mu}^{s}(\gamma) \leq \bar{\mu}^{s}(\alpha)+\bar{\mu}^{s}(\beta) .
$$

If we move a segment in a $\mathscr{G}^{u}$ leaf by holonomy along $\mathscr{G}^{s}$ leaves to another segment in a $\mathscr{G}^{u}$ leaf, the two segments will have the same value under $\bar{\mu}^{s}$.

Our goal is to show that there exists $\rho>0$ such that for any segment $\alpha$ in a $\mathscr{G}^{u}$ leaf $\bar{\mu}^{s}(\alpha)=\rho \nu^{s}(\alpha)$. We will use the fact that $g$ has a Markov partition $\mathscr{R}=$ $\left\{R_{1}, \ldots, R_{n}\right\}$ - see [FLP, Exposé $10 \S \mathrm{IV}, \S \mathrm{V}$ and $\S \mathrm{VI}$ ] for the definition and properties of Markov partitions.

Denote by $S_{i}^{u} \subset R_{i}$ any segment in a $\mathscr{G}^{u}$ leaf which goes across $R_{i}$. The values $a_{i}=\nu^{s}\left(S_{i}^{u}\right)$ and $b_{i}=\bar{\mu}^{s}\left(S_{i}^{u}\right)$ do not depend on the choice of $S_{i}^{u}$ because any two such choices differ by holonomy along $\mathscr{G}^{s}$ leaves. Call $x_{i j}$ the number of times $g^{-1}\left(R_{j}\right)$ crosses the interior of $R_{i}$. This gives a positive matrix $X=\left(x_{i j}\right)_{1 \leq i, j \leq n}$. From [FLP, Exposé 10 Lemma 1 p. 205], the matrix $X$ has power which is strictly positive. Moreover, if $X^{l}=\left(x_{i j}^{(l)}\right)_{1 \leq i, j \leq n}$, then $\left(x_{i j}^{(l)}\right)_{1 \leq i, j \leq n}$ is the number of times that $f^{-l}\left(R_{j}\right)$ crosses $\boldsymbol{R}_{\boldsymbol{i}}$. It follows from this remark and (2) above that, if we define $A$ (resp. $B$ ) as the vector with components $a_{1}, \ldots, a_{n}$ (resp. $b_{1}, \ldots, b_{n}$ ), then we have:

$$
\forall l \geq 1, \lambda^{l} A=X^{l} A \text { and } \lambda^{l} B \leq X^{l} B .
$$

Call $\tilde{a}_{1}, \ldots, \tilde{a}_{n}$ the components of the eigenvector with eigenvalue $\lambda$ of the transpose of $X$ normalized by the relation $\sum_{i=1}^{n} \tilde{a}_{i} a_{i}=1$. From Perron-Frobenius theory, it is well known that $\tilde{a}_{i}>0$ and $\lim _{l \rightarrow \infty} \lambda^{-l} X^{\prime} B=\rho A$, with $\rho=\sum_{i=1}^{n} \tilde{a}_{i} b_{i}$. It follows from (3) that $B \leq \rho A$. If one of the components of the last inequality was strict multiplying the $i$-component by the strictly positive $\tilde{a}_{i}$ and summing would give $\rho=\sum_{i=1}^{n} \tilde{a}_{i} b_{i}<$ $\rho \sum_{i=1}^{n} \tilde{a}_{i} a_{i}=\rho$ which is impossible. So we have:

$$
\forall i=1, \ldots, n, \quad b_{i}=\rho a_{i} .
$$

Suppose now that $\alpha$ is a segment in a $\mathscr{G}^{u}$ leaf. For each $j=1, \ldots, n$ and each $l \geq 1$, call $\alpha_{j}^{l}$ the number of $\mathscr{G}^{u}$ segments intersecting $\alpha$ which are contained in $f^{-l}\left(R_{i}\right)$ and go across it. Using (1) through (4), we obtain:

$$
\bar{\mu}^{s}(\alpha) \leq \sum_{i=1}^{n} \alpha_{j}^{l} \lambda^{-l} b_{j}=\sum_{i=1}^{n} \alpha_{j}^{l} \rho \lambda^{-l} a_{j} \rightarrow \rho \nu^{s}(\alpha)
$$

This shows the inequality $\bar{\mu}^{s}(\alpha) \leq \rho \nu^{s}(\alpha)$. Note now that $\rho$ cannot be zero, because that would imply that the image of $\varphi(N)$ would be contained in one leaf of $\mathscr{F}^{s}$ and hence, by the $g$ invariance, it would be reduced to one point. Suppose that some segment $\beta$ in a $\mathscr{G}^{u}$ leaf verifies $\bar{\mu}^{s}(\beta)<\rho \nu^{s}(\beta)$. Choose a point $x$ in the interior of $\beta$ and call $x_{\infty}$ an accumulation point of the sequence $g^{-l}(x), l \geq 0$. It is clear that the unstable leaf through $x_{\infty}$ contains arbitrarily small segments $\alpha$, containing $x_{\infty}$, and such that $\bar{\mu}^{s}(\alpha)<\rho \nu^{s}(\alpha)$. The density of the stable leaf through $x_{\infty}$ and the invariance by holonomy show that the same strict inequality is true for any segment in a $\mathscr{G}^{u}$ leaf. This is absurd, since we have equality for any segment in a $\mathscr{G}^{u}$ leaf contained in a $R_{i}$ and going across it.

By rescaling $\nu^{s}$, we can assume that $\rho=1$. This allows us to interpret what we obtained in the following way: the map $\varphi$ is an isometry of any non singular leaf 
of $\mathscr{G}^{u}$ endowed with the metric defined by $\nu^{s}$ onto the corresponding leaf of $\mathscr{F}^{u}$ endowed with the metric defined by $\mu^{s}$. Of course, the same result can be obtained with stable foliations. It is now easy to finish the proof of the lemma.

Acknowledgements. I am most grateful to Mike Handel not only for his active help in writing this paper, but also for the constant stimulation provided by his work. My debt to his papers [H1, H2] is enormous. The proof I am giving for Theorem 1 was found in Spring 1986, while I was enjoying the hospitality of the Mathematics Institute at the University of Warwick for the 'Special year in smooth ergodic theory'.

\section{REFERENCES}

[CP] E. M. Coven \& M. E. Paul. Endomorphisms of irreducible subshifts of finite type. Math. Sys. Th. 8 (1974-75), 167-175.

[FLP] A. Fathi, F. Laudenbach \& V. Poenaru. Travaux de Thurston sur les surfaces. Astérisque 66-67 (1979).

[F] J. Franks. Anosov diffeomorphisms. Proc. Symp. in Pure Mathematics 14 (1970), 61-94.

[H1] M. Handel. Global shadowing of pseudo-Anosov homeomorphisms. Ergod. Th. \& Dynam. Sys. 5 (1985), 373-377.

[H2] M. Handel. Entropy and semi-conjugacy in dimension two. Ergod. Th. \& Dynam. Sys. 8 (1988), 585-596.

[L] J. Lewowicz. Persistence in expansive systems. Ergod. Th. \& Dynam. Sys. 3 (1983), 567-578.

[MS] J. Morgan \& P. B. Shalen. Valuations, trees, and degenerations of hyperbolic structures: I. Ann. Math. 120 (1984), 401-476. 